\title{
Avaliação da sintomatologia depressiva de mulheres no climatério com a escala de rastreamento populacional para depressão CES-D
}

\author{
Depressive symptoms in climacteric women evaluated by the Center for \\ Epidemiological Studies Depression Scale
}

\author{
Rita de Cássia Leite Fernandes ${ }^{1}$, Marcia Rozenthal ${ }^{2}$ \\ ${ }^{1}$ Mestre. Médica, Ministério da Saúde. ${ }^{2}$ Doutora. Professora adjunta, Escola de Medicina e Cirurgia, Universidade Federal do Estado do Rio de \\ Janeiro (UNIRIO), Rio de Janeiro, RJ. \\ Este estudo foi realizado no Instituto Fernandes Figueira, Fundação Oswaldo Cruz (FIOCRUZ), Rio de Janeiro, RJ.
}

\section{Resumo}

Introdução: $\mathrm{O}$ objetivo deste estudo foi avaliar a sintomatologia depressiva em mulheres climatéricas com a escala de depressão CES-D (Center for Epidemiological Studies Depression Scale), do National Institute of Mental Health (EUA).

Método: Estudo transversal com 151 mulheres entre 40 e 65 anos de idade, usuárias de serviço de ginecologia geral em unidade de atenção básica à saúde no Rio de Janeiro. Aplicou-se a escala CES-D e um questionário estruturado para a obtenção de dados sociodemográficos, clínicos e ginecológicos. O nível de corte $>15$ pontos na CES-D foi considerado como indicativo de quadro depressivo.

Resultados: A média de pontuação da amostra foi de 9,2 pontos (desvio padrão $=9,0$ ). Os itens mais pontuados da escala foram relativos à insônia, tristeza e desânimo. Não houve associação significativa entre os escores e o período climatérico, características sociodemográficas, clínicas ou ginecológicas, exceto para as mulheres com presença de sintomas psíquicos, histórico depressivo pregresso e uso atual de antidepressivos $(\mathrm{p}=0,000)$. Entre as 32 mulheres $(21 \%)$ com pontuação $>15$ na CES-D, $72 \%$ referiram episódio depressivo pregresso. Dentre as participantes sem histórico depressivo, as perimenopáusicas apresentaram escores $>15$ com maior freqüência.

Conclusão: Essa casuística, oriunda de serviço não-especializado em menopausa ou saúde mental, revelou baixas pontuações médias na escala de sintomas depressivos CES-D, e o item insônia foi o mais pontuado. O histórico de depressão foi fator de associação com a alta pontuação na escala, mas não a fase climatérica em que a mulher se encontrava. A maior freqüência de pontuação acima do nível de corte nas mulheres sem histórico depressivo pregresso que estavam na perimenopausa sugere a maior vulnerabilidade, nessa fase, a episódios depressivos novos.

Descritores: Climatério, menopausa, depressão.

\begin{abstract}
Introduction: The objective of this study was to evaluate depressive symptoms in climacteric women using the Center for Epidemiological Studies Depression Scale (CES-D) from the National Institute of Mental Health (USA).

Method: This is a cross-sectional study carried out in a gynecological outpatient unit in Rio de Janeiro, Brazil, including 151 climacteric women between 40 and 65 years of age. The CES-D was used to assess depressive symptoms and a structured interview obtained sociodemographic, clinical and gynecological data. A score above 15 points on the CES-D was considered as a cut-point for depressive state.

Results: Mean CES-D score was 9.2 points (standard deviation $=9.0$ ). Insomnia, sadness and despondency had the highest scores. There was no significant association between CES-D scores and the climacteric period, sociodemographic, clinical or gynecological characteristics, except for women with psychiatric symptoms, history of depression or on antidepressants $(\mathrm{p}=0.000)$. In 32 women
\end{abstract}

\section{Correspondência:}

Rita de Cássia Leite Fernandes, Rua Marquês de Abrantes, 171/502, Flamengo, CEP 22230-060, Rio de Janeiro, RJ.

Não há conflitos de interesse associados à publicação deste artigo.

Copyright (C) Revista de Psiquiatria do Rio Grande do Sul - SPRS 
(21\%) who scored $>15$ on the CES-D, $72 \%$ had already suffered from a depressive state. Women with no history of depressive disorder scored more frequently above 15 when they were perimenopausal.

Conclusion: This sample of climacteric women, from a non-specialized mental or menopausal health service, had low mean scores on the CES-D, with the item insomnia being the most highly scored. History of a previous depressive episode, but not the woman's climacteric period, was a risk factor for higher scores on the CES-D. In the group of women with no history of depression, the perimenopausal women had more scores above the cut-point. This fact may suggest that the perimenopause is a period of higher susceptibility to new onset of depressive episodes.

Keywords: Climacteric, menopause, depression.

\section{Introdução}

Alterações do humor são queixas relatadas com grande freqüência aos especialistas de clínicas de menopausa, mas ainda existe controvérsia sobre a existência de associação entre declínio da função ovariana e saúde mental da mulher ${ }^{1,2}$. Uma terminologia psiquiátrica antiga designava a depressão que acompanha algumas mulheres na menopausa como "melancolia involutiva". Entretanto, outros estressores, característicos desse período da vida, podem contribuir para os quadros depressivos climatéricos, e não apenas a senescência ou o declínio hormonal. Estudos comunitários apontam que a sintomatologia depressiva em mulheres na meia-idade está associada a fatores como atitude em relação à menopausa, saúde geral da mulher, ansiedade, problemas de relacionamento, presença de outros sintomas climatéricos e histórico de episódio depressivo anterior ${ }^{2-4}$.

Segundo definição da Organização Mundial de Saúde ${ }^{5}$, menopausa é a última menstruação, que ocorre em média aos 50 anos de idade, e só pode ser reconhecida após 12 meses de amenorréia. O climatério delimita a passagem da vida reprodutiva para a nãoreprodutiva, iniciando-se por volta dos 40 anos e terminando na sexta década, para a maioria das mulheres. O seu principal aspecto biológico é a redução da produção dos hormônios esteróides sexuais pelos folículos ovarianos, principalmente estrogênio e progesterona. A perimenopausa é o período que se inicia com as primeiras irregularidades menstruais, em média aos 45 anos, e se estende até 1 ano após a menopausa. Durante a perimenopausa, ocorrem flutuações dos níveis séricos hormonais e maior freqüência dos sintomas vasomotores como fogachos e sudorese noturna. $\mathrm{O}$ aumento da freqüência dos transtornos do humor nessa fase continua em debate ${ }^{2,4,6,7}$.

As altas taxas de sintomas depressivos obtidas em pesquisas realizadas em clínicas especializadas em menopausa ou em saúde mental ${ }^{8-11}$ não se confirmaram em alguns estudos de base comunitária. Sugeriu-se que a perimenopausa pode ser período de maior risco para os distúrbios do humor, mas não necessariamente representa um momento de risco para a depressão maior ${ }^{12,13}$. Em 2000, Woods et al..$^{14}$ apresentaram dados do estudo populacional sobre saúde da mulher realizado em Seattle (WA, EUA), onde 508 mulheres foram acompanhadas anualmente com a escala de depressão do Centro de Estudos Epidemiológicos (Center for Epidemiological Studies Depression Scale - CES-D). O padrão mais prevalente foi o de baixa pontuação ao longo do tempo, que corresponde a uma baixa intensidade de sintomas depressivos. Estudo recente de Freeman et al. ${ }^{4}$ avaliou, ao longo de 8 anos do climatério, um grupo de 231 mulheres comunitárias com a CES-D. À medida que elas entravam na perimenopausa, metade apresentou sintomas depressivos significativos e 59 desenvolveram depressão maior. O estudo concluiu que a transição da perimenopausa para a menopausa se associa a um aumento de cinco vezes no risco de depressão.

No Brasil, estudos em clínicas especializadas também indicam alta prevalência de sintomatologia depressiva nas mulheres climatéricas. De Lorenzi et al. ${ }^{3}$ encontraram $73,2 \%$ de melancolia ou tristeza utilizando o índice menopausal de Kupperman, um dos instrumentos mais utilizados por ginecologistas para avaliação da sintomatologia climatérica. Em amostra oriunda de serviços de saúde mental e de menopausa, Soares ${ }^{15}$ encontrou 49,5\% de sintomas depressivos intensos com o Self Report Questionnaire (SRQ-20). Recentemente, Veras et al. ${ }^{16}$ apontaram prevalências significativas de transtornos ansiosos $(34,9 \%)$ e de depressão maior (31,4\%) no ambulatório de menopausa de uma instituição de ensino no Rio de Janeiro, utilizando o Mini International Neuropsychiatric Interview (MINI).

Fazer o diagnóstico de depressão e medir a gravidade dos sintomas depressivos relatados pelos indivíduos são tarefas distintas. As chamadas escalas de rastreio constituem um meio simples e rápido de identificar indivíduos supostamente afetados ou vulneráveis à depressão. $\mathrm{O}$ nível de pontuação numa escala aponta para a presença da síndrome depressiva, apesar de não ser adequada para categorizar o paciente dentro de um diagnóstico nosológico de depressão. A medida dos sintomas depressivos por meio de escalas de sintomas é tanto um recurso de auxílio ao clínico quanto um meio utilizado em pesquisas que avaliam características depressivas na população.

A escala de rastreamento populacional de depressão CES-D, do National Institute of Mental 
Health (EUA) visa a identificar sintomas depressivos em estudos populacionais ${ }^{17,18}$. A sua utilidade estendese à investigação das relações entre sintomas depressivos e variáveis demográficas e psicossociais e à identificação de fatores de risco. Entre os brasileiros, a escala nunca foi utilizada com o propósito de investigar sintomatologia depressiva no climatério, tendo sido traduzida e validada por Silveira \& Jorge ${ }^{17}$ em estudo de adolescentes e por Tavares ${ }^{19}$, que estudou, em idosos, as características psicométricas da CES-D em relação à GDS (Geriatric Depression Scale). Utilizando o nível de corte de pontuação $>15$ pontos na CES-D, essa autora encontrou boa consistência interna para a escala $($ alpha $=0,86)$, com altos índices de sensibilidade $(74,6 \%)$ e especificidade $(73,6 \%)$ para a detecção dos casos de depressão que tinham sido identificados pela GDS. Algumas características da CES-D podem torná-la um bom instrumento de aferição de sintomatologia depressiva nas mulheres climatéricas, pela simplicidade de aplicação e investigação tanto de sintomas depressivos cognitivos, como somáticos, psicomotores e relativos a dificuldades nas relações interpessoais.

O objetivo principal da presente pesquisa foi descrever a pontuação de um grupo de mulheres climatéricas, usuárias de serviço de atenção básica à saúde, na escala CES-D. O objetivo secundário foi verificar a existência de associação entre as médias de pontuação na escala de depressão e as características sociodemográficas, ginecológicas e clínicas da amostra.

\section{Método}

Trata-se de um estudo transversal, realizado entre janeiro e maio de 2007 com usuárias do ambulatório de ginecologia geral da Policlínica Antônio Ribeiro Netto (PARN), convidadas a participar pela ordem de chegada à recepção. $\mathrm{O}$ critério de inclusão foi idade entre 40 e 65 anos, período que, segundo a OMS, inclui mulheres do início do declínio da atividade folicular ovariana até a homeostase hormonal ${ }^{5}$. Esta pesquisa faz parte de um estudo sobre cognição no climatério ${ }^{20}$ e, por isso, o critério de exclusão foi histórico atual ou pregresso de acidente vascular cerebral. A pesquisa foi aprovada pelo Comitê de Ética em Pesquisa da Secretaria Municipal de Saúde do Rio de Janeiro. A amostra foi constituída por 151 mulheres que consentiram em participar e que assinaram o termo de consentimento livre e esclarecido.

Os dados sociodemográficos analisados foram idade, escolaridade, renda familiar per capita e situação conjugal. Os dados ginecológicos registrados foram: características atuais do ciclo menstrual, número de gestações, número de filhos vivos, cirurgias ginecológicas e uso de reposição hormonal. As participantes que não tiveram alteração do seu padrão menstrual no último ano foram consideradas prémenopáusicas; as que referiram ciclos irregulares, mais longos ou mais curtos, ou amenorréia de menos de 12 meses de duração foram classificadas como perimenopáusicas, e as que não menstruavam há mais de um ano, como pós-menopáusicas. Foi considerada como portadora da condição clínica a participante que, na aplicação de um questionário estruturado, relatou a presença de diabetes melito, hipertensão arterial, dislipidemias e doenças neurológicas e psiquiátricas. $\mathrm{O}$ índice de massa corporal (IMC) foi calculado dividindo-se o peso (em $\mathrm{kg}$ ) pelo quadrado da altura da mulher (em m). Questionou-se a presença dos sintomas: nervosismo, irritabilidade, depressão ou insônia, e a resposta positiva a qualquer desses itens foi considerada positiva para sintomas psíquicos. O histórico de episódios depressivos prévios foi avaliado pela pergunta: "A senhora já teve depressão anteriormente ou tomou remédios para depressão?".

A escala de depressão CES-D foi aplicada pela pesquisadora a todas as participantes. Ela é composta por 20 itens que questionam sintomas depressivos nos últimos 7 dias anteriores à entrevista (Tabela 1). Cada resposta admite quatro gradações crescentes de intensidade (nunca ou raramente, às vezes, freqüentemente e sempre) e pontuações correspondentes $(0,1,2$ e 3$)$. Os itens $4,8,12$ e 16 (positivos) são pontuados com gradação inversa. $\mathrm{O}$ escore final varia de 0 a 60 pontos e corresponde à soma da pontuação de todas as respostas. Os itens da CES-D incluem questões relativas ao humor (itens $3,4,6,8,9,10,12,16,17$ e 18), sintomas psicossomáticos (itens 1, 5 e 11), sintomas ligados às interações sociais (itens 14,15 e 19) e sintomas relacionados à iniciativa motora (itens 2,7 , 13 e 20). Utilizamos a pontuação $>15$ pontos na escala como nível de corte que indicaria a presença de sintomas depressivos significativos ${ }^{4,17,19}$. As médias de pontuação foram comparadas entre os subgrupos climatéricos e foram analisadas as características das mulheres com pontuação acima do nível de corte.

Os dados coletados foram registrados e analisados no programa Epi-Info ${ }^{\circledR}$, versão 3.3 .2 (2005). As variáveis categóricas foram avaliadas quanto a sua freqüência. As variáveis contínuas com distribuição normal foram comparadas através da análise de variância (ANOVA) e as não-paramétricas, através do teste de Kruskal-Wallis, calculando-se suas médias e desvios padrão. Utilizamos a regressão linear com cálculo do coeficiente de correlação de Pearson (r) para a verificação da associação entre variáveis. $O$ nível de significância adotado para todos os testes estatísticos foi de $5 \%(\mathrm{p}<0,05)$. 
Tabela 1 - Escala de rastreamento populacional para depressão CES-D*

\begin{tabular}{|c|c|c|c|c|c|}
\hline Durante a última semana: & $\begin{array}{c}\text { Raramente } \\
(<1 \mathrm{dia}) \\
0 \text { ponto }\end{array}$ & $\begin{array}{c}\text { Durante pouco } \\
\text { tempo } \\
\text { (1 ou } 2 \text { dias) } \\
1 \text { ponto }\end{array}$ & $\begin{array}{c}\text { Durante tempo } \\
\text { moderado } \\
\text { ( } 3 \text { ou } 4 \text { dias) } \\
2 \text { pontos }\end{array}$ & $\begin{array}{c}\text { Durante maior } \\
\text { parte do tempo } \\
\text { (5 a } 7 \text { dias }) \\
3 \text { pontos }\end{array}$ & Total \\
\hline
\end{tabular}

1) Senti-me incomodado com coisas que habitualmente não me incomodam

2) Não tive vontade de comer, tive pouco apetite

3) Senti não conseguir melhorar meu estado de ânimo, mesmo com ajuda de familiares e amigos

4) Senti-me, comparando-me às outras pessoas, tendo tanto valor quanto a maioria delas

5) Senti dificuldade em me concentrar no que estava fazendo

6) Senti-me deprimido

7) Senti que tive de fazer esforço para dar conta das minhas tarefas habituais

8) Senti-me otimista em relação ao futuro ${ }^{\dagger}$

9) Considerei que a minha vida tinha sido um fracasso

10) Senti-me amedrontado

11) Meu sono não foi repousante

12) Estive feliz

13) Falei menos que o habitual

14) Senti-me sozinho

15) As pessoas não foram amistosas comigo

16) Aproveitei minha vida ${ }^{\dagger}$

17) Tive crises de choro

18) Senti-me triste

19) Senti queas pessoas não gostavam de mim

20) Não consegui levar adiante minhas coisas

Total

CES-D = escala de depressão do Centro de Estudos Epidemiológicos (Center for Epidemiological Studies Depression Scale). * Versão brasileira de Silveira \& Jorge ${ }^{17}$.

† Nesses itens a pontuação deve ser inversa.

\section{Resultados}

As participantes da pesquisa possuíam, em média, 51,8 anos de idade (desvio padrão - DP $=6,6$ ), 6,8 anos de escolaridade ( $\mathrm{DP}=3,8$ ), com $6 \%$ de analfabetas, e renda mensal per capita de 1,3 salários mínimos $(\mathrm{DP}=$ $0,8)$ (Tabela 2). A maior parte possuía algum tipo de renda própria e um cônjuge. Entre as 39 mulheres (26\%) que haviam utilizado reposição hormonal, 2/3 delas o fez por período inferior a um ano. Verificou-se um elevado número de portadoras de uma ou mais doenças metabólicas ou cardiovasculares crônicas, destacandose sobrepeso e obesidade (IMC > 25) e hipertensão arterial. 
Tabela 2 - Características sociodemográficas, ginecológicas e clínicas da amostra de mulheres climatéricas da Policlínica Antonio Ribeiro Netto, Rio de Janeiro, $2007(\mathrm{n}=151)$

\begin{tabular}{|c|c|c|c|c|c|}
\hline \multirow[t]{2}{*}{ Característica } & \multirow{2}{*}{$\begin{array}{c}\text { Total } \\
n=151 \\
(100 \%)\end{array}$} & \multicolumn{4}{|c|}{ Período climatérico } \\
\hline & & $\begin{array}{c}\text { Ignorado* } \\
\mathrm{n}=15(9,9 \%)\end{array}$ & $\begin{array}{c}\text { Pré-menopausa } \\
n=33(24,3 \%)\end{array}$ & $\begin{array}{l}\text { Perimenopausa } \\
n=31(22,8 \%)\end{array}$ & $\begin{array}{c}\text { Pós-menopausa } \\
n=72(52,9 \%)\end{array}$ \\
\hline Idade - anos, média \pm DP & $51,8 \pm 6,6$ & $54,0 \pm 5,4$ & $44,2 \pm 3,0$ & $48,3 \pm 3,6$ & $56,4 \pm 4,9$ \\
\hline Escolaridade- anos, média \pm DP & $6,8 \pm 3,8$ & $7,1 \pm 4,8$ & $7,8 \pm 3,6$ & $6,7 \pm 3,7$ & $6,2 \pm 3,8$ \\
\hline $\begin{array}{l}\text { Renda - } \mathrm{n}^{\mathrm{o}} \text { sal. mínimos, média } \pm \\
\text { DP }\end{array}$ & $1,3 \pm 0,8$ & $1,7 \pm 1,2$ & $1,1 \pm 0,6$ & $1,2 \pm 0,7$ & $1,3 \pm 0,8$ \\
\hline Com cônjuge, n (\%) & $95(63)$ & $10(67)$ & $21(64)$ & $20(65)$ & $44(61)$ \\
\hline Com renda, n (\%) & $102(67)$ & $12(80)$ & $26(79)$ & $16(52)$ & $48(67)$ \\
\hline TRH, n (\%) & $39(26)$ & $8(53)$ & 0 & $1(3,2)$ & $30(42)$ \\
\hline Diabetes melito, $\mathrm{n}(\%)$ & $12(7,9)$ & $9(6,6)$ & $3(9,1)$ & $3(9,7)$ & $3(4,2)$ \\
\hline Hipertensão arterial, n (\%) & $57(38)$ & $8(53)$ & $8(24)$ & $8(26)$ & $33(46)$ \\
\hline Dislipidemia, n (\%) & $55(36)$ & $9(60)$ & $7(21)$ & $7(23)$ & $32(44)$ \\
\hline $\mathrm{IMC}^{\dagger}>25, \mathrm{n}(\%)$ & $90(63)$ & $11(73)$ & $14(44)$ & $23(77)$ & $42(65)$ \\
\hline Epilepsia, n (\%) & $3(2,0)$ & $1(6,7)$ & 0 & 0 & $2(2,8)$ \\
\hline Sintomas psíquicos, n (\%) & $31(20)$ & $1(7)$ & $7(21)$ & $5(16)$ & $18(25)$ \\
\hline Histórico depressivo, n (\%) & $51(34)$ & $6(40)$ & $14(42)$ & $9(29)$ & $22(31)$ \\
\hline Uso de ansiolítico, n (\%) & $18(12)$ & 0 & $4(12)$ & $3(9,7)$ & $11(15)$ \\
\hline Uso de antidepressivo, $\mathrm{n}(\%)$ & $16(11)$ & $1(6,7)$ & $2(6,1)$ & $3(9,7)$ & $10(14)$ \\
\hline Uso de neuroléptico, n (\%) & $1(0,7)$ & 0 & $1(3,0)$ & 0 & 0 \\
\hline Tabagismo, n (\%) & $15(10)$ & 0 & $6(18)$ & $1(3,2)$ & $8(11)$ \\
\hline Etilismo, n (\%) & $4(2,6)$ & 0 & 0 & 0 & $4(5,6)$ \\
\hline
\end{tabular}

$\mathrm{DP}=$ desvio padrão; ICM = índice de massa corporal; TRH = terapia de reposição hormonal.

* Histerectomizadas.

† Índice de massa corporal: em nove participantes não foi possível obter os dados antropométricos $(\mathrm{n}=142)$.

Cerca de $20 \%$ das mulheres climatéricas relataram a presença de algum sintoma psíquico atual e $34 \%$ referiram episódio depressivo anterior. No momento da pesquisa, $12 \%$ das participantes utilizavam ansiolíticos e $11 \%$ antidepressivos; uma mulher que referia ser portadora de esquizofrenia utilizava neuroléptico $(0,7 \%)$. A única doença neurológica relatada foi epilepsia, que acometia três participantes $(2 \%)$. O tabagismo foi referido por $10 \%$, e o etilismo freqüente ( $>1$ vez por semana), por apenas $2,6 \%$.

O período climatérico era desconhecido em 15 participantes por terem realizado histerectomia previamente. Dentre as não histerectomizadas $(n=136)$, um pouco mais da metade da amostra encontrava-se na pós-menopausa (53\%), $23 \%$ na perimenopausa e $24 \%$ na pré-menopausa. $\mathrm{O}$ grupo das mulheres mais jovens, pré-menopáusicas, exibiu a maior escolaridade. Hipertensão arterial e dislipidemias foram mais freqüentes entre as pós-menopáusicas. A freqüência de sintomas psíquicos atuais também foi maior no grupo pós-menopáusico, mas o histórico depressivo pregresso estava mais presente no relato do grupo pré- menopáusico. O consumo de ansiolíticos e/ou antidepressivos exibiu tendência ao aumento na pósmenopausa. Não houve diferenças significativas em relação ao consumo de álcool entre as diferentes fases do climatério e nas histerectomizadas. O tabagismo foi referido por $10 \%$ da amostra, sendo as mulheres perimenopáusicas e histerectomizadas as que apresentaram o menor índice.

A pontuação média da casuística na CES-D foi de $9,2$ pontos ( $\mathrm{DP}=9,0)$, com mínimo de 0 e máximo de 50 pontos. O nível de escolaridade exibiu fraca associação positiva com a presença de sintomas depressivos, com índice de correlação de Pearson (r) igual a 0,14 . Também houve pequena associação negativa entre a pontuação na CES-D e a idade da participante, com $r=-0,1$. As demais variáveis sociodemográficas e clínico-ginecológicas não tiveram associação significativa com a pontuação na escala CES$\mathrm{D}$, exceto por escores significativamente maiores entre as mulheres com presença de sintomas psíquicos em relação às que não referiram esses sintomas (médias 14,9 versus 7,7 respectivamente; $p=0,001$ ), entre as com 
histórico depressivo pregresso em relação às que não relataram depressão anterior (médias 14,0 versus 6,8 respectivamente; $p=0,000$ ) e entre as que usam antidepressivos em relação às que não usaram (médias 18,5 versus 8,1 respectivamente; $p=0,000$ ).

A análise dos escores médios na CES-D em função do período climatérico conhecido não evidenciou diferenças estatisticamente significativas $(\mathrm{p}=0,741)$, situando-se $<10$ pontos em todos os grupos (Tabela 3). A menor pontuação média foi obtida pelas mulheres pós-menopáusicas. Um total de 32 mulheres apresentou escores $>15$ pontos na escala de depressão CES-D, representando $21,2 \%$ da amostra. A sua distribuição dentro dos grupos climatéricos conhecidos foi semelhante: $21 \%$ das prémenopáusicas, $26 \%$ das perimenopáusicas e $18 \%$ das pós-menopáusicas tiveram essa pontuação acima do ponto de corte $(\mathrm{p}=0,945)$.

Tabela 3 - Escores obtidos por mulheres climatéricas na escala de depressão CES-D, segundo período climatérico $(\mathrm{n}=151)$

\begin{tabular}{lcc}
\hline Período climatérico & $\begin{array}{c}\text { Pontuação CES-D } \\
\text { Média } \pm \text { DP }\end{array}$ & $\begin{array}{c}\text { Freqüência CES-D }>\mathbf{1 5} \\
\mathbf{n}(\boldsymbol{\%})\end{array}$ \\
\hline Total $(\mathrm{n}=151)$ & $9,23 \pm 9,04$ & $32(21,2)$ \\
Ignorado* $(\mathrm{n}=15)$ & $8,73 \pm 6,25$ & $4(26,6)$ \\
Pré-menopausa $(\mathrm{n}=33)$ & $9,93 \pm 9,91$ & $7(21,2)$ \\
Perimenopausa $(\mathrm{n}=31)$ & $9,96 \pm 9,15$ & $8(25,8)$ \\
Pós-menopausa $(\mathrm{n}=72)$ & $8,70 \pm 9,19$ & $13(18,0)$ \\
\hline
\end{tabular}

CES-D = escala de depressão do Centro de Estudos Epidemiológicos (Center for Epidemiological Studies Depression Scale); DP = desvio padrão.

* Histerectomizadas.

Os dois grupos com escores abaixo e acima de 15 pontos na escala de sintomas depressivos não apresentaram diferenças significativas nas médias de idade e escolaridade. Os demais fatores sociodemográficos e clínico-ginecológicos analisados também não tiveram freqüências de ocorrência diferentes entre os grupos. As únicas exceções foram as maiores freqüências de presença de sintomas psíquicos e de histórico depressivo pregresso nas mulheres do grupo com pontuação $>15$ pontos: 37,5 e $71,9 \%$, respectivamente. Dentre as mulheres com pontuação acima do nível de corte na escala de depressão (>15), observou-se que a menor freqüência de histórico depressivo pregresso foi obtida entre as mulheres na perimenopausa (Tabela 4), ou seja, para $62,5 \%$ das perimenopáusicas com pontuação alta na CES-D, este poderia representar o primeiro episódio depressivo.

Tabela 4 - Freqüência de histórico depressivo pregresso na amostra de mulheres climatéricas com pontuação $>15$ na escala CES-D, segundo período climatérico $(\mathrm{n}=32)$

\begin{tabular}{lcc}
\hline Período climatérico & \multicolumn{2}{c}{ Pontuação CES-D > 15 } \\
\cline { 2 - 3 } & $\begin{array}{c}\text { Com histórico depressivo anterior } \\
\mathbf{n}(\%)\end{array}$ & $\begin{array}{c}\text { Sem histórico depressivo anterior } \\
\text { n (\%) }\end{array}$ \\
\hline Ignorado* $(\mathrm{n}=4)$ & $3(75,0)$ & $1(25,0)$ \\
Pré-menopausa $(\mathrm{n}=7)$ & $6(85,7)$ & $1(14,3)$ \\
Perimenopausa $(\mathrm{n}=8)$ & $3(37,5)$ & $5(62,5)$ \\
Pós-menopausa $(\mathrm{n}=13)$ & $11(84,6)$ & $2(15,4)$ \\
\hline
\end{tabular}

CES-D = escala de depressão do Centro de Estudos Epidemiológicos (Center for Epidemiological Studies Depression Scale).

* Histerectomizadas. 
A análise da pontuação da casuística nas 20 questões da escala, evidenciou que o maior escore médio foi alcançado pelo item 11 (Meu sono não foi repousante), seguido por dois itens relativos ao humor, números 18 (Senti-me triste) e 3 (Senti não conseguir melhorar meu estado de ânimo mesmo com a ajuda de familiares e amigos). Dentro do grupo de mulheres com pontuação > 15 na CES-D, o item com maior média de pontos foi o número 18 , seguido pelos números 6 (Sentime deprimido) e 11. No grupo com $>15$ pontos na escala de depressão, o item com maior média de pontuação foi relativo ao sono, número 11, seguido pelos do humor, números 18 e 3 .

\section{Discussão}

A presente pesquisa encontrou pontuação média de 9,2 pontos $(\mathrm{DP}=9,0)$ na escala de depressão CES-D entre mulheres climatéricas. Não foram detectadas associações significativas entre o escore obtido na escala e o período climatérico em que a participante se encontrava (pré-menopausa, perimenopausa ou pósmenopausa). Esse achado é concordante com o estudo longitudinal de Woods et al. ${ }^{14}$, que, utilizando a mesma escala, não encontraram evidências de aumento dos sintomas depressivos em função das diferentes fases climatéricas.

A maioria dos fatores sociodemográficos e clínicoginecológicos analisados também não apresentou associação com os escores da CES-D na amostra. Não houve associação significativa entre a intensidade de sintomas depressivos e a idade, renda, estado conjugal, escolaridade, paridade, uso de terapia de reposição hormonal, número de gestações ou de filhos vivos e a presença de diabetes, hipertensão arterial, dislipidemias ou sobrepeso/obesidade. Como seria esperado, as mulheres que referiram sintomas psíquicos atuais, uso atual de antidepressivo e episódio depressivo anterior tiveram pontuação significativamente mais alta. Outros autores já relataram o histórico depressivo pregresso como fator de risco importante para sintomatologia depressiva no climatério ${ }^{1-4,9,11,13}$.

Durante o período reprodutivo da mulher (entre a menarca e a menopausa), ocorrem três momentos de aumento da chance de diagnóstico de depressão maior: na menarca, na gravidez e na perimenopausa ${ }^{1,21,22}$. Postula-se a existência de uma associação entre as alterações do humor e o ambiente hormonal, principalmente ditado pelo eixo hipotálamo-hipófisetireóide e pelos efeitos cerebrais dos hormônios sexuais, que influenciariam, em conjunto, a resposta do cérebro humano ao meio ambiente $21,23-25$.

As modulações do humor e o aumento da freqüência de depressão associados a determinados períodos de alterações endógenas dos esteróides ovarianos e ao uso de contraceptivos orais favorecem a hipótese do efeito direto desses hormônios na regulação da atividade monoaminérgica central ${ }^{21,24,25}$. As evidências da ação dos esteróides sexuais sobre a fisiologia cerebral provêm de pesquisas básicas em laboratório, de estudos populacionais e de ensaios clínicos. As pesquisas em animais demonstraram a existência de receptores estrogênicos em regiões corticais e cerebelares, no hipocampo, no hipotálamo, no sistema límbico e na amígdala ${ }^{22,24}$. Pela sua ligação a receptores intracelulares, os hormônios sexuais exercem efeitos genômicos que incluem a modificação da seqüência de transcrição de genes que regulam a síntese e o metabolismo de neurotransmissores e que modulam os receptores do fator de crescimento neural. A sua ação não-genômica se dá em nível da membrana celular, permitindo a modulação de sistemas que regulam a serotonina (down-regulation dos receptores 5- $\mathrm{HT}_{2}$ ), a noradrenalina ou a dopamina ${ }^{24,26}$.

As fases folicular e ovulatória do ciclo menstrual, caracterizadas pelo pico dos níveis de estrogênio e hormônio luteinizante, se correlacionam com maior sensação de bem-estar, enquanto os sintomas de tensão e depressão aumentam no período prémenstrual, quando os níveis dos estrógenos declinam $^{1,21}$. Algumas mulheres, em torno de 5\%, experimentam depressão pré-menstrual cíclica forte o suficiente para preencher os critérios de transtorno disfórico pré-menstrual) ${ }^{1}$. A gravidez e o puerpério também são acompanhados de maior incidência de quadros depressivos, e ensaios clínicos demonstraram melhora da depressão pós-parto com terapia estrogênica ${ }^{2}$. O uso do 17- estradiol transdérmico para o tratamento de quadros depressivos perimenopáusicos também obteve sucesso quando utilizado isoladamente ou em combinação com inibidores de recaptação da serotonina, tendo sido considerado um efeito independente da melhora dos sintomas vasomotores ${ }^{7,15,25,27}$. Esses achados reforçam a teoria de uma associação entre as flutuações dos níveis dos esteróides gonadais no organismo feminino e os transtornos do humor ${ }^{1,23}$.

Na presente pesquisa, não houve associação entre a pontuação $>15$ na escala CES-D, obtida por $21 \%$ da amostra, e os fatores sociodemográficos e clínicoginecológicos analisados, mas o histórico depressivo estava presente em grande parcela desse grupo (72\%). Tal achado foi concordante com vários autores que sugeriram que diferentes fatores podem influenciar o aparecimento de sintomas depressivos climatéricos, entre eles o histórico depressivo pregresso ${ }^{1-4,9,11,13}$. Entre autores nacionais, Soares ${ }^{15}$ investigou mulheres em clínica especializada de menopausa com o SRQ-20, e encontrou $49,5 \%$ de sintomatologia depressiva 
significativa. A análise psiquiátrica subseqüente dessas pacientes revelou que $60 \%$ delas tinha diagnóstico de episódio depressivo maior. Estudo domiciliar realizado em Campinas ${ }^{28}$ revelou alta prevalência de sintomas psicológicos ( $82 \%$ de nervosismo), avaliados por intermédio de questionário estruturado, cuja intensidade ou freqüência não se correlacionaram apenas ao estado menopausal, mas também a fatores psicossociais. De Lorenzi et al. ${ }^{3}$ investigaram a sintomatologia climatérica em 254 pacientes atendidas em ambulatório de climatério e encontraram como sintomas mais prevalentes a irritabilidade $(87,1 \%)$, as artralgias e mialgias $(77,5 \%)$ e a melancolia e tristeza $(73,2 \%)$, utilizando o índice menopausal de Kupperman. Também encontraram associação entre a intensidade dessa sintomatologia e fatores como atividade física, tabagismo, IMC, número de gestações e atitudes em relação à menopausa. Nas mulheres com percepção da menopausa como evento vantajoso, os sintomas psicológicos foram menos intensos, ao passo que naquelas com atitudes negativas em relação à menopausa, eles foram significativamente mais intensos.

A análise dos escores da amostra da presente pesquisa nos subitens da escala CES-D evidenciou que o distúrbio do sono foi o problema mais pontuado, seguido de tristeza e desânimo, mantendo-se nessa posição mesmo quando se subtraem do cálculo as mulheres com pontuação $>15$ na escala. Tal achado se alinha àqueles encontrados em estudos sobre sintomatologia climatérica em que a irritabilidade, tristeza, insônia e os fogachos situam-se entre as queixas mais prevalentes $3,6,14,27,28$.

Em geral, os estudos que encontraram altas prevalências de transtornos depressivos no climatério são oriundos de clínicas especializadas em saúde mental ou menopausa, e poderiam estar enviesados por uma clientela naturalmente mais sintomática. Recentemente, contudo, o estudo americano de Freeman et al. ${ }^{4}$ acompanhou mulheres comunitárias sem histórico depressivo desde o pré-climatério até a menopausa. Esse trabalho encontrou aumento da chance de um diagnóstico de depressão quando a mulher entra na perimenopausa (razão de chance $=$ 2,50). O estudo, em virtude de seu desenho metodológico, que ajustou fatores de confundimento, pesquisou mulheres oriundas da comunidade em seleção aleatória, usou como critério de exclusão o diagnóstico prévio de episódio depressivo, acompanhou os níveis séricos hormonais e analisou a evolução das participantes (tendo como referencial o seu próprio desempenho antes do início da perimenopausa), reforçou a teoria da maior vulnerabilidade das mulheres aos sintomas depressivos e à depressão maior na perimenopausa.
A análise da casuística do presente estudo, dividida por períodos climatéricos conhecidos, evidenciou 32 mulheres com $>15$ pontos na CES-D (21\%), com freqüências semelhantes dentro dos períodos pré-menopausa, perimenopausa e pósmenopausa. Quando essa pontuação foi analisada em relação ao histórico depressivo anterior, observouse que mais de $62 \%$ das perimenopáusicas tinham histórico negativo, podendo ser considerados possíveis episódios depressivos novos. Tal dado coincide com os supracitados resultados de Freeman et al. ${ }^{4}$, em que o maior risco de depressão nova foi encontrado na perimenopausa.

Em resumo, os dados da literatura sugerem que a presença de sintomatologia depressiva em algumas mulheres no período de transição da vida reprodutiva para a não-reprodutiva origina-se de uma constelação de fatores socioculturais, individuais e biológicos que, atuando em conjunto, favoreceriam o aparecimento das flutuações do humor e da depressão.

Algumas limitações do presente estudo devem ser apontadas. Idealmente, o climatério deveria ser caracterizado por dosagens hormonais. Reconhece-se que a seleção das mulheres apenas pelo critério cronológico pode ter incluído algumas que ainda não apresentavam declínio da função ovariana. Esta pesquisa, entretanto, baseou-se em estudos sistematizados no relatório da Organização Mundial de Saúde ${ }^{5}$, que indicam que a grande maioria das mulheres inicia a redução insidiosa da produção de estrógenos ovarianos no início da quarta década de vida, atingindo níveis insignificantes e inativação de receptores hormonais por volta dos 65 anos. Outras duas questões também devem ser apontadas: a avaliação de episódios depressivos anteriores sem a utilização de instrumento diagnóstico padronizado pode ter enviesado o resultado derivado dessa variável; e o pequeno número de participantes nas diferentes fases do climatério entre as que tiveram pontuação acima do nível de corte na escala CES-D pode ter determinado um poder estatístico insuficiente para detectar diferenças entre os subgrupos. Contudo, a investigação das associações existentes entre a pontuação na escala com o histórico depressivo e o período climatérico não era o objetivo principal do estudo, e os dados foram apresentados com o intuito de contribuir para estudos futuros, com indicações de tendências que devem ser confirmadas com uma aferição mais bem definida da variável sobre histórico depressivo pregresso e amostra numericamente superior. Ainda que os presentes resultados não sejam representativos do universo total de mulheres no climatério, podem estar próximos de representar aquelas que freqüentam o tipo de instituição utilizada no estudo, uma unidade pública básica de saúde. 


\section{Conclusão}

No presente estudo, foram encontradas baixas pontuações médias de mulheres climatéricas na escala de rastreamento populacional para depressão CES-D, concordantes com outros estudos efetuados em clínicas não especializadas. Não houve associação entre a intensidade de sintomas depressivos com os fatores sociodemográficos, ginecológicos e clínicos analisados, exceto pela previsível maior pontuação alcançada por aquelas com histórico depressivo pregresso e mais queixas psíquicas. Cerca de $20 \%$ da amostra atingiu > 15 pontos na escala CES-D, sugerindo uma prevalência de depressão nesta amostra climatérica próxima àquela obtida para a população feminina em geral. No grupo das que pontuaram acima do nível de corte, $72 \%$ já tinham sofrido de depressão, sugerindo que o histórico depressivo pregresso é um dos fatores de risco para a presença de sintomatologia depressiva nesse período da vida da mulher. Mais de $60 \%$ das participantes que se encontravam no período da perimenopausa e que atingiram $>15$ pontos na CES-D negavam histórico depressivo anterior, resultado que se alinharia com a hipótese de aumento da vulnerabilidade ao primeiro episódio depressivo nessa fase específica do climatério, já apontado por outros autores.

\section{Referências}

1. Dennerstein L. Mood and menopause. 2nd. World Congress on Women's Mental Health [conference coverage on line] Apr 2004. http://www.medscape.com/viewarticle/473294

2. Soares CN, Cohen LS. The perimenopause, depressive disorders, and hormonal variability. São Paulo Med J. 2001;119(2):78-83.

3. De Lorenzi DRS, Danelon C, Saciloto B, Padilha Jr. I. Fatores indicadores da sintomatologia climatérica. Rev Bras Ginecol Obstet. 2005;27(1):12-9.

4. Freeman EW, Sammel MD, Lin H, Nelson DB. Associations of hormones and menopausal status with depressed mood in women with no history of depression. Arch Gen Psychiatry. 2006;63(4):375-82.

5. World Health Organization (WHO). Research on the menopause in the 1990s. Geneva: WHO; 1996. WHO Technical Report Series 866.

6. Novaes C, Almeida OP, de Melo NR. Mental health among perimenopausal women attending a menopause clinic: possible association with premenstrual syndrome? Climacteric. 1998;1(4):264-70.

7. Soares CN, Almeida OP, Joffe H, Cohen LS. Efficacy of estradiol for the treatment of depressive disorders in perimenopausal women. Arch Gen Psychiatry. 2001;58(6):529-34.

8. Schmidt PJ, Rubinow DR. Menopause-related affective disorders: a justification for further study. Am J Psychiatry. 1991;148(7):84452.

9. Kaufert PA, Gilbert P, Tate R. The Manitoba Project: a reexamination of the link between menopause and depression. Maturitas. 1992;14(2):143-55.

10. Stewart DE, Boydell K, Derzko C, Marshall V. Psychologic distress during the menopausal years in women attending a menopause clinic. Int J Psychiatry Med. 1992;22(3):213-20.

11. Hay AG, Bancroft J, Johnstone EC. Affective symptoms in women attending a menopause clinic. Br J Psychiatry. 1994;164(4):513-6.

12. Hunter M. The south-east England longitudinal study of the climacteric and post-menopause. Maturitas. 1992;14(2):117-26.

13. Avis NE, Brambilla D, McKinlay SM, Vass K. A longitudinal analysis of the association between menopause and depression. Results from the Massachusetts Women's Health Study. Ann Epidemiol. 1994;4(3):214-20.

14. Woods NF, Mitchell ES, Adams C. Memory Functioning Among Midlife Women: Observations from the Seattle Midlife Women's Health Study. Menopause. 2000;7(4):257-65.

15. Soares CN. Depressão na perimenopausa: morbidade psiquiátrica, associação com distúrbios perimenstruais e impacto da terapêutica com 17 b-estradiol [tese]. São Paulo: Universidade de São Paulo; 1999.

16. Veras AB, Rassi A, Valença AM, Nardi AE. Prevalência de transtornos depressivos e ansiosos em uma amostra ambulatorial brasileira de mulheres na menopausa. Rev Psiquiatr RS. 2006;28(2):27-38

17. Silveira DX, Jorge MR. Propriedades psicométricas da escala de rastreamento populacional para depressão CES-D em populações clínica e não-clínica de adolescentes e adultos jovens. Rev Psiquiatr Clin. 1998;25(5):251-61.

18. Radloff LS. The CES-D Scale: A Self-report Depression Scale for Research in the General Population. Appl Psychol Measurement. 1977;1(3):385-401.

19. Tavares SS. Sintomas depressivos entre idosos: relações com classe, mobilidade e suporte social percebidos e experiência de eventos estressantes [dissertação]. São Paulo: Faculdade de Educação da Universidade Estadual de Campinas; 2004.

20. Fernandes RCL. Climatério e cognição: desempenho de um grupo de mulheres climatéricas no mini-exame do estado mental e no teste de memória da lista de palavras [dissertação]. Rio de Janeiro: Instituto Fernandes Figueira; 2007.

21. Almeida OP. Sex playing with the mind. Arq Neuropsiquiatr. 1999;57(3A):701-6.

22. Sherwin BB. Estrogen and cognitive functioning in women. Endocr Rev. 2003;24(2):133-51.

23. Kuehner C. Gender differences in unipolar depression: an update of epidemiological findings and possible explanations. Acta Psychiatr Scand. 2003;108(3):163-74.

24. McEwen BS. Clinical review 108: The molecular and neuroanatomical basis for estrogen effects in the central nervous system. J Clin Endocrinol Metab. 1999;84(6):1790-7.

25. Schmidt PJ. Depression, the perimenopause, and estrogen therapy. Ann N Y Acad Sci 2005;1052:27-40.

26. Veiga S, García-Segura LM, Azcoitia I. Propriedades neuroprotectoras de los esteróides sexuales y los neuroesteroides. Rev Neurol. 2004;39(11):1043-51.

27. Soares CN, Prouty J, Poitras J. Ocorrência e tratamento de quadros depressivos por hormônios sexuais. Rev Bras Psiquiatr. 2002; 24(Supl 1):48-54.

28. Pedro AO, Pinto-Neto AM, Costa-Paiva L, Osis MJ, Hardy E. Procura de serviço médico por mulheres climatéricas brasileiras. Rev Saude Publica. 2002;36(4):484-90. 